\title{
Autophagie rénale
}

\section{Nicolas Pallet}

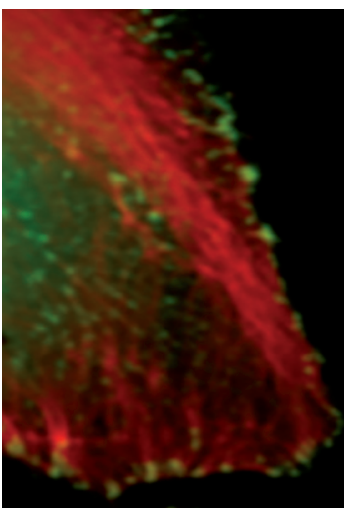

Inserm U1147,

Université Paris Descartes,

45, rue des Saints Pères,

75006 Paris, France.

nicolas.pallet@parisdescartes.fr impliquée dans le maintien de l'homéostasie cellulaire lors de stress micro-environnementaux, a des propriétés anti-inflammatoires, et module l'immunité adaptative. L'autophagie est aussi impliquée dans la physiologie, la pathologie, et le vieillissement rénal; elle module la réponse tissulaire aux agressions aiguës; elle joue un rôle majeur dans le maintien de l'homéostasie podocytaire et tubulaire, et réduit la sévérité des lésions liées au vieillissement. Les effets néphroprotecteurs podocytaires et épithéliaux de l'autophagie sont liés à l'élimination des mitochondries altérées qui activent les inflammasomes et l'apoptose, ainsi qu'à la clairance des agrégats protéiques. Sur le plan translationnel, l'autophagie est sans conteste une piste de recherche majeure pour le développement de thérapeutiques néphroprotectrices et l'identification de nouveaux biomarqueurs de lésions tissulaires rénales. <

\section{Les maladies rénales}

Toute altération de la structure du parenchyme rénal pourra avoir des conséquences biologiques et cliniques avec un impact significatif en termes de morbi-mortalité. La maladie rénale chronique (MRC) touche des millions de personnes à travers le monde. Elle constitue donc un enjeu de santé publique majeur en France et dans le

Vignette (Photo $\odot$ Inserm-Corinne Antignac).

monde. La destruction définitive des éléments fonctionnels élémentaires qui constituent les reins, les néphrons, aboutit à une perte de fonction qui peut conduire à une insuffisance rénale définie par une baisse du débit de filtration glomérulaire (marqueur clé d'évaluation de la fonction rénale). La morbi-mortalité associée à la MRC est considérable avec un sur-risque de mortalité de 2 à 5 fois supérieur à celui de la population générale. Schématiquement, la MRC est la conséquence de deux grands types de stress tissulaire : des stress aigus (acute kidney injury, AKI, ou insuffisance rénale aiguë, IRA) et des stress chroniques, tous deux à l'origine de lésions structurelles irréversibles. L'IRA, qui peut survenir à la suite de toxicités aiguës, médicamenteuses ou après hypoperfusion tissulaire (survenant lors d'un choc par exemple), génère des lésions néphroniques, en particulier de l'épithélium tubulaire, dont la restitution ad integrum n'est pas systématique. II est en effet maintenant bien montré qu'après une IRA, la réparation tissulaire s'accompagne de processus pro-fibrosants et pro-inflammatoires qui vont générer des altérations structurelles définitives, aboutissant à la MRC [1]. Les stress chroniques à l'origine d'une MRC sont liés aux conséquences, notamment ischémiques, des lésions microvasculaires et capillaires, associées par exemple à l'hypertension artérielle et au diabète, affectant en particulier le parenchyme rénal. Les lésions de MRC s'auto-amplifient, indépendamment de la cause initiale. En effet, la fibrose interstitielle et la raréfaction capillaire (liée à la destruction du réseau capillaire) altèrent la diffusion de l'oxygène et des nutriments aux cellules tubulaires conduisant à leur mort et/ou leur dédifférenciation. La réduction néphronique, secondaire aux maladies rénales, induit une hyperfiltration au niveau des glomérules encore sains qui est délétère à terme en induisant un stress des podocytes ${ }^{1}$ et, in fine, leur apoptose. Cette perte podocytaire provoque des lésions de hyalinose segmentaire et focale secondaire (ou HSF) ${ }^{2}$, et de glomérulosclérose. Enfin, la protéinurie produite par

\footnotetext{
${ }^{1}$ Les podocytes entourent les cellules glomérulaires et participent à la constitution du filtre glomérulaire. ${ }^{2}$ La HSF est une atteinte glomérulaire caractérisée par des lésions focales qui favorisent la sclérose glomérulaire.
} 
ces lésions glomérulaires exerce une toxicité sur l'épithélium tubulaire. Schématiquement, deux régions du néphron, l'épithélium tubulaire et le glomérule, constituent des cibles privilégiées pour le stress tissulaire rénal et le développement de lésions de fibrose interstitielle, d'atrophie tubulaire et de glomérulosclérose constituent les signatures de la MRC.

\section{Le stress de l'épithélium tubulaire}

De nombreuses situations peuvent compromettre la perfusion rénale et aboutir à une ischémie tissulaire: un choc, une ischémie froide liée aux prélèvements d'organes, des médicaments vasoconstricteurs, des pathologies artérielles aiguës et chroniques. Physiologiquement, les zones profondes, dites médullaires, présentent une pression faible en oxygène. Elles sont donc très sensibles aux variations de perfusion sanguine rénale. L'activité cellulaire de réabsorption de solutés est particulièrement importante au niveau de certains types cellulaires comme l'épithélium tubulaire rénal proximal. Elle est très coûteuse sur le plan énergétique. Ce coût est supporté par un pool mitochondrial considérable qui assure la production d'ATP nécessaire et, in fine, le fonctionnement adéquat des pompes $\mathrm{Na}^{+} / \mathrm{K}^{+}$ATPases qui produisent les gradients ioniques nécessaires au transfert des solutés de l'urine au travers de la cellule vers les vasa recta (les vaisseaux droits qui cheminent le long des anses de Henlé) qui longent les tubules. Certaines zones de l'épithélium tubulaire sont ainsi très sensibles à la survenue d'une ischémie. Elles seront le siège, le cas échéant, des lésions de nécrose tubulaire aiguë (NTA) qui se caractérisent par une desquamation de l'épithélium consécutive à la mort des cellules par apoptose et/ou par nécrose. Il est maintenant admis que le processus de la régénération épithéliale s'accompagne de fibrogenèse qui laisse ainsi une mémoire de l'agression dans le parenchyme rénal. Des épisodes d'IRA répétés peuvent générer à terme des lésions histologiques qui résulteront en une MRC. À côté des épisodes ischémiques pourvoyeurs d'IRA, des origines néphrotoxiques peuvent participer également à la survenue de lésions de nécrose tubulaire aiguë. Quelles qu'en soient les causes, il apparaît évident que les processus cellulaires d'adaptation aux stress micro-environnementaux qui sont activés dans les cellules tubulaires jouent un rôle important pour le maintien de l'intégrité structurelle et fonctionnelle des néphrons. Les mécanismes mis en jeu dans l'autophagie semblent des acteurs majeurs de ce processus.

\section{Stress podocytaire}

L'intégrité parfaite de la barrière de filtration glomérulaire est nécessaire pour un maintien de l'imperméabilité aux protéines qui soit quasi absolue: l'excrétion physiologique de protéines est en effet de l'ordre de 0,20 grammes par 24 heures alors que, dans cette même période, environ $10 \mathrm{~kg}$ de protéines transitent par les capillaires glomérulaires. La barrière de filtration glomérulaire est constituée d'un endothélium fenêtré qui repose sur une membrane basale complexe. Composée de chaînes protéiques chargées négativement (collagène de type IV, héparane sulfates, etc.), elle limite le passage des protéines de haute masse moléculaire en imposant des contraintes de taille et de charge. Le versant urinaire de la barrière est constitué de podocytes (des cellules parvenues à un stade terminal de différenciation et qui n'ont, classiquement, aucune capacité de prolifération ni de régénération). Toute altération de l'intégrité de ces cellules peut donc être à l'origine de conséquences significatives sur la physiologie glomérulaire et aboutir à des séquelles irréversibles. Les podocytes morts se détachent de la barrière de filtration glomérulaire et sont éliminés dans l'urine primitive. La dénudation de la barrière qui en résulte va conduire à la formation de lésions de HSF et, en conséquence, de sclérose glomérulaire définitive. Les glomérules sains restants sont alors soumis à des pressions accrues qui favorisent de nouveau un stress podocytaire et la mort des cellules, à l'origine d'un cercle vicieux. De nombreuses situations physiologiques (vieillissement) ou pathologiques (maladies auto-immunes, diabète, etc.) peuvent être à l'origine d'un stress podocytaire. Plusieurs mécanismes adaptatifs dont l'autophagie ont été récemment décrits pour leur implication dans le maintien de l'intégrité cellulaire et de la barrière de filtration glomérulaire.

\section{Systèmes d'adaptation au stress cellulaire}

Le néphron, et en particulier les glomérules et les tubules, peuvent être considérés comme les senseurs ou intégrateurs de nombreux stress micro-environnementaux d'origines variées qui vont aboutir, au niveau de la cellule, à la mise en place de systèmes d'adaptation moléculaire afin d'y faire face. Les plus importants d'entre eux (et qui interagissent) impliquent I'HIF-l $\alpha$ (hypoxia-inducible factor 1 alpha), mTOR (mammalian target of rapamycin), la réponse au stress UPR (unfolded protein response) et I'autophagie. Ils ont en commun de pouvoir détecter les fluctuations du micro-environnement qui, lorsqu'elles sont trop importantes, constituent un danger pour la cellule à l'origine du stress. Ainsi, la régulation de la protéostasie réalisée par l'intermédiaire de ces différents intégrateurs tient une place importante dans les processus de réponse aux stress rénaux [2]. Néanmoins, tous ces processus régulateurs sont également plus ou moins impliqués dans des réponses de type inflammatoire et dans le remodelage tissulaire, notamment par le biais de la synthèse de la matrice extracellulaire et la production de cytokines pro-fibrosantes (Figure 1). Parmi ces processus adaptatifs, l'autophagie joue un rôle capital dans le maintien de l'intégrité structurelle et fonctionnelle du néphron. 


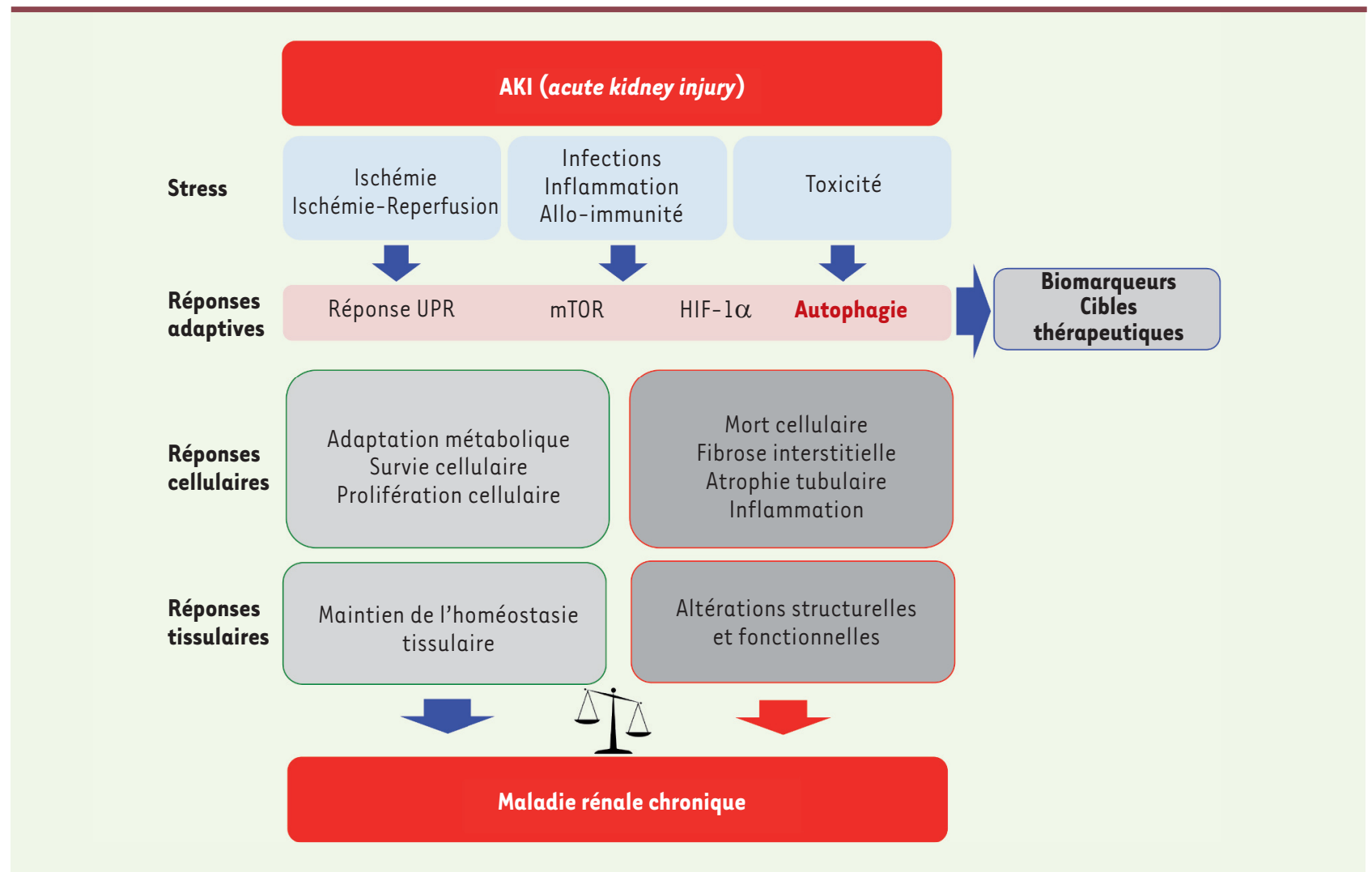

Figure 1. Rôle des voies d'adaptation au stress cellulaire dans le maintien de l'homéostasie tissulaire rénale. De nombreuses situations aboutissant à une insuffisance rénale aiguë génèrent un stress cellulaire. En réponse à ce stress, des voies biologiques, dont l'autophagie, vont être activées. Ces processus ont en général des fonctions cytoprotectrices, permettant une restauration structurelle et fonctionnelle, mais peuvent activer en parallèle des processus pro-fibrosants et pro-inflammatoires, qui peuvent être délétères. HIF-l $\alpha$ : hypoxia-inducible factor 1 alpha; mTOR : mammalian target of rapamycin; UPR : unfolded protein response.

\section{Autophagie rénale}

Les premières investigations portant sur l'autophagie dans l'homéostasie rénale ont été réalisées au cours des années 1970 et 1980, mais ces études restaient assez confidentielles et seule l'autophagie des tubules proximaux était examinée. Les principaux axes de recherche portaient alors sur des études ultrastructurales (avec la caractérisation des structures impliquées dans le processus autophagique et le développement de méthodes permettant leur détection) [3-7], sur les processus impliqués dans sa régulation, notamment par l'insuline, les œstrogènes ou les diurétiques [8-10], et enfin sur les fonctions métaboliques de l'autophagie [11, 12]. Ces travaux ont mis en évidence les notions fondamentales caractérisant l'autophagie, qui sont la dégradation de protéines et de mitochondries (très présentes dans le tubule proximal), la modulation du processus, et le lien fort existant avec le métabolisme énergétique de la cellule. L'explosion des connaissances portant sur les fonctions et la régulation de l'autophagie dans le cadre de la physiologie rénale date d'une dizaine d'années. Elles ont été facilitées par l'apparition de modèles murins génétiquement modifiés et invalidés pour des protéines de régulation clé de l'autophagie. II faut néanmoins noter qu'à l'heure actuelle, il n'existe pas de mala- die rénale, en particulier génétique, liée à un déficit d'autophagie; en d'autres termes, le rôle de l'autophagie dans les maladies rénales n'est en effet déduit que de modèles murins invalidés et exposés à des stress tissulaires, le corollaire en pathologie humaine n'étant pas établi.

\section{Autophagie glomérulaire}

Toutes les cellules constituant les glomérules sont impliquées dans la physiopathologie des maladies rénales et un rôle important de l'autophagie a été décrit principalement dans les podocytes, mais également, dans une moindre mesure, dans les cellules endothéliales et les cellules mésangiales. Les podocytes sont les cellules les plus fragiles des glomérules. Dans de nombreuses pathologies humaines, la perte podocytaire est directement liée à la sévérité et au pronostic de la maladie. Les facteurs biologiques impliqués dans cette perte des podocytes sont multiples: le stress du réticulum endoplasmique (RE), le 


\section{Podocytes}

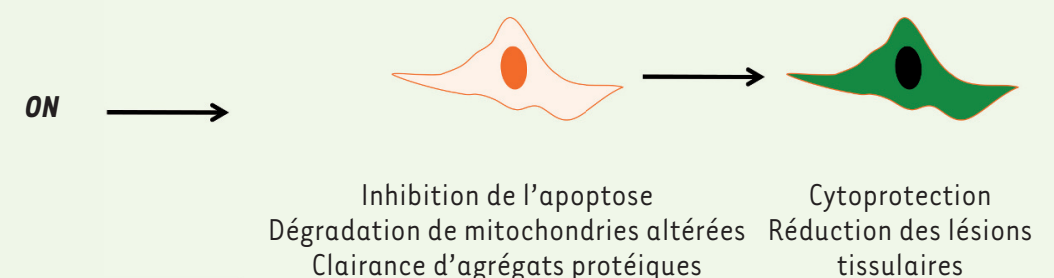

Vieillissement

Stress podocytaire

Adriamycine

Puromycine

Hyperglycémie

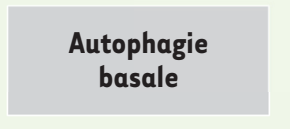

Clairance d'agrégats protéiques

\begin{abstract}
Cytoprotection
duction des lésions
tissulaires
\end{abstract}

OFF

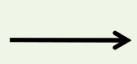

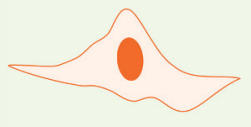

Apoptose

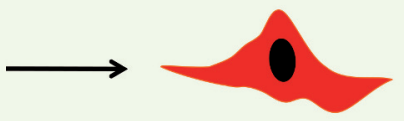

Perte podocytaire Glomérulosclérose Protéinurie

Figure 2. Fonctions de l'autophagie dans les podocytes. Le niveau de base du flux autophagique est augmenté dans les podocytes. Un déficit en autophagie produit une perte podocytaire accélérée durant le vieillissement et une moindre tolérance au stress. Les modèles murins dans lesquels l'autophagie ne peut être induite développent des lésions de hyalinose segmentaire et focale. Le mécanisme de perte podocytaire (par apoptose ?) n'est pas établi et, de fait, l'impact de l'autophagie sur ce processus n'est pas définitivement expliqué.

stress oxydatif ou l'accumulation de mitochondries altérées [13-15]. Les glomérules sont particulièrement sensibles au vieillissement, et les podocytes, ne pouvant se régénérer, ont développé des processus permettant l'élimination des agrégats protéiques et des organelles altérées, qui sont très actifs et participent au maintien de la viabilité de ces cellules au cours du vieillissement (Figure 2). Le niveau d'autophagie basal est élevé dans les podocytes. L'invalidation des gènes de l'autophagie Atg5 ou Atg7 au niveau des podocytes est responsable de lésions de glomérulosclérose chez la souris âgée qui sont associées à des altérations cellulaires typiques du vieillissement (accumulation de lipofuscine, de protéines oxydées, et d'agrégats ubiquitinylés et positifs pour le séquestosome 1, ou p62) [16]. Le déficit podocytaire en protéines régulatrices de l'autophagie accroît donc la susceptibilité aux maladies rénales. Plus spécifiquement, il favorise le développement accéléré de lésions glomérulaires en lien avec ces pathologies pour aboutir à des lésions de HSF [17-19] qui constituent la cicatrice évolutive de la perte podocytaire et conduisent à la MRC [20]. L'inhibition du flux autophagique podocytaire, ou sa réduction, favorise l'accumulation d'agrégats protéiques et de mitochondries altérées. Ceux-ci activent la voie de signalisation NF-KB (nuclear factor-kappa B) et l'inflammasome NLRP3 (NOD-like receptor family, pyrin domain containing 3 ) mais également un stress du réticulum endoplasmique, un stress oxydatif et la mort cellulaire. L'autophagie est également impliquée dans la physiopathologie de lésions glomérulaires induites par les dépôts de complexes immuns, en particulier la néphrite lupique. Un rôle majeur de l'autophagie dans la biologie des cellules $B$ a en effet été montré dans le modèle de souris lupiques chez lesquelles le gène Atg5 a été invalidé dans les lymphocytes B : ces souris développent moins de lésions glomérulaires que les souris sauvages [21].

La néphropathie diabétique est une cause majeure d'insuffisance rénale dans les pays industrialisés. L'hypertrophie, l'effacement des pédicelles (des ramifications provenant des podocytes qui s'entrelacent autour des capillaires glomérulaires), et le détachement podocytaire sont des caractéristiques de la néphropathie diabétique. Des données récentes ont montré le rôle central de l'autophagie dans la survenue des lésions glomérulaires associées à cette pathologie. $\varepsilon n$ effet, l'hyperglycémie est un facteur activateur du flux autophagique qui a une fonction cytoprotectrice. Cependant, une hyperglycémie chronique s'accompagne d'une réduction du flux autophagique et d'une activation, en parallèle, de la voie mTOR résultant en une hypertrophie podocytaire qui est délétère [22, 23]. L'activation de mTOR, et des voies anaboliques qui lui sont reliées, favorisent en effet l'émergence des lésions podocytaires (en lien avec l'accumulation d'agrégats protéiques et d'organelles altérées) et de glomérulosclérose. L'interruption du flux autophagique dans ces podocytes accélère la survenue des lésions glomérulaires [24, 25], alors que la rapamycine, le resveratrol, ou la restriction calorique qui l'induisent, ont des effets bénéfiques sur la podocytopathie et la glomérulosclérose dans les modèles murins de néphropathie diabétique [26-29]. Plus directement, l'observation que 
les souris déficientes en $\operatorname{Atg} 5$, au niveau systémique ou uniquement dans les podocytes, développent une podocytopathie accélérée dans des modèles de diabètes de type I et II a permis de montrer l'implication de l'autophagie dans le maintien de l'intégrité des cellules [30]. L'activation du système rénine-angiotensine, et la production d'angiotensine $2^{3}$, contribuent au développement des MRC, à la fois vasculaires et glomérulaires. En particulier, l'angiotensine 2 joue un rôle important dans la physiopathologie de la néphropathie diabétique notamment en favorisant la perméabilité glomérulaire, en augmentant la pression intraglomérulaire, mais aussi en induisant l'apoptose des podocytes, leur détachement, et in fine, le développement d'une glomérulosclérose. In vitro, l'angiotensine 2 active le flux autophagique en induisant la production d'espèces réactives de l'oxygène, mais sa contribution précise au développement de la néphropathie diabétique reste à déterminer [31]. Ces données soulignent néanmoins le rôle du stress oxydatif dans le développement de la podocytopathie associée à la glomérulopathie diabétique. L'hème oxygénase, produite dans les podocytes en réponse à des concentrations élevées de glucose, contribue au maintien de la viabilité cellulaire en induisant l'autophagie [32]. Au cours de la néphropathie diabétique, l'endothélium capillaire glomérulaire est également affecté. Dans ce cas également, l'autophagie est requise pour limiter le stress cellulaire induit par I'hyperglycémie [33].

Les cellules mésangiales sont des péricytes (cellules contractiles localisées dans le mésangium) qui permettent un support structural et fonctionnel aux glomérules. Elles permettent la synthèse de la matrice extracellulaire qui constitue le mésangium. Elles sont la cible de nombreux stimulus pathologiques, tels que les produits de glycation avancée $(A G E)$ au cours de la néphropathie diabétique, qui induisent leur prolifération et une synthèse excessive de matrice extracellulaire. Le transforming growth factor $\beta$ (TGF $\beta$ ), une cytokine profibrosante ayant un rôle important dans la physiopathologie de la glomérulosclérose, induit l'autophagie dans les cellules mésangiales réduisant la production de matrice mésangiale en initiant la dégradation de collagène de type I, un effet a priori bénéfique de l'autophagie dans le processus [34]. Le flux autophagique peut également être activé dans les cellules mésangiales en réponse à la production d'AGE, mais les conséquences de ce processus sur le développement de la néphropathie diabétique restent inconnues [35].

\section{Autophagie tubulaire}

L'importance de l'autophagie dans l'évolution et la sévérité du processus lésionnel conduisant à une IRA a été évaluée dans de nombreux modèles de stress tissulaires aigus. Il est maintenant admis, encore une fois grâce à l'utilisation de modèles murins où différents gènes ont été invalidés, que l'autophagie joue un rôle protecteur, à la fois pour le maintien de la viabilité cellulaire au moment de l'agression, mais également dans la conservation de la structure néphronique au décours

\footnotetext{
${ }^{3}$ L'angiotensine 2 exerce un rôle dans la régulation de la pression artérielle et de la volémie (volume
} sanguin circulant) par le biais du système rénine-angiotensine. de l'épisode (Figure 3). Ces propriétés protectrices de l'autophagie sont retrouvées dans un grand éventail de stress : néphrotoxicité, ischémie-reperfusion, sepsis, ou infections urinaires [36]. Même si la perte de l'autophagie dans le tubule rénal (examinée en utilisant des souris n'exprimant pas ATG5 ou ATG7 dans l'épithélium tubulaire et soumises à une ischémie-reperfusion, ou une néphrotoxicité) conduit à l'augmentation de la mortalité cellulaire, et à une aggravation des lésions de NTA, le mécanisme précis de protection mis en jeu avec I'autophagie dans ces situations précises reste obscur. Les données disponibles impliquent cependant l'élimination de mitochondries altérées qui sont génératrices de stress oxydant, d'inflammation (par l'intermédiaire des inflammasomes) et d'apoptose [36, 37].

Un deuxième cadre physiopathologique au cours duquel l'autophagie semble jouer un rôle est celui de la fibrose interstitielle et de l'atrophie tubulaire, des lésions séquellaires non spécifiques qui surviennent à la suite d'une IRA, caractéristiques de la MRC et du vieillissement tissulaire. La ligature de l'uretère est le modèle le plus utilisé pour étudier les processus de fibrogenèse rénale. Bien que traduisant probablement mal la complexité du processus conduisant à la fibrose, il s'avère que le flux autophagique est activé dans ce modèle [38]. Appliqué à des souris déficientes en autophagie (délétion homozygote de $L C 3$, ou haplo-insuffisance en Beclin-1) dans l'épithélium tubulaire, les lésions de fibrose interstitielle se révèlent plus sévères, indiquant le rôle protecteur de l'autophagie. Un des mécanismes proposés repose sur le rôle du TGF $\beta[39,40]$. Les relations entre autophagie et TGF $\beta$ sont complexes: le TGF $\beta$ induit l'activation du flux autophagique dans les cellules tubulaires proximales en culture par un mécanisme qui dépend de la production d'espèces réactives de l'oxygène. Ainsi activée, l'autophagie permettrait l'élimination des protéines oxydées et des mitochondries altérées. L'autophagie est également impliquée dans la protéolyse directe du TGF $\beta$ mature. En effet, des souris invalidées pour les gènes codant LC3 ou Beclin-1 ont des niveaux de production de TGF $\beta$ mature qui sont plus élevés que ceux observés chez les souris sauvages. Ces souris développent également des lésions de fibrose interstitielle plus sévères [34].

La LC3-associated phagocytosis (LAP) est un processus de phagocytose impliquant la protéine LC3 dans le transfert du matériel du phagosome vers le site de dégradation lysosomal. Ce mécanisme, émergeant, pourrait permettre de comprendre le lien entre IRA et MRC. II a en effet été montré que les corps apoptotiques tubulaires, qui sont produits lors d'une NTA, étaient phagocytés par les cellules tubulaires rénales 


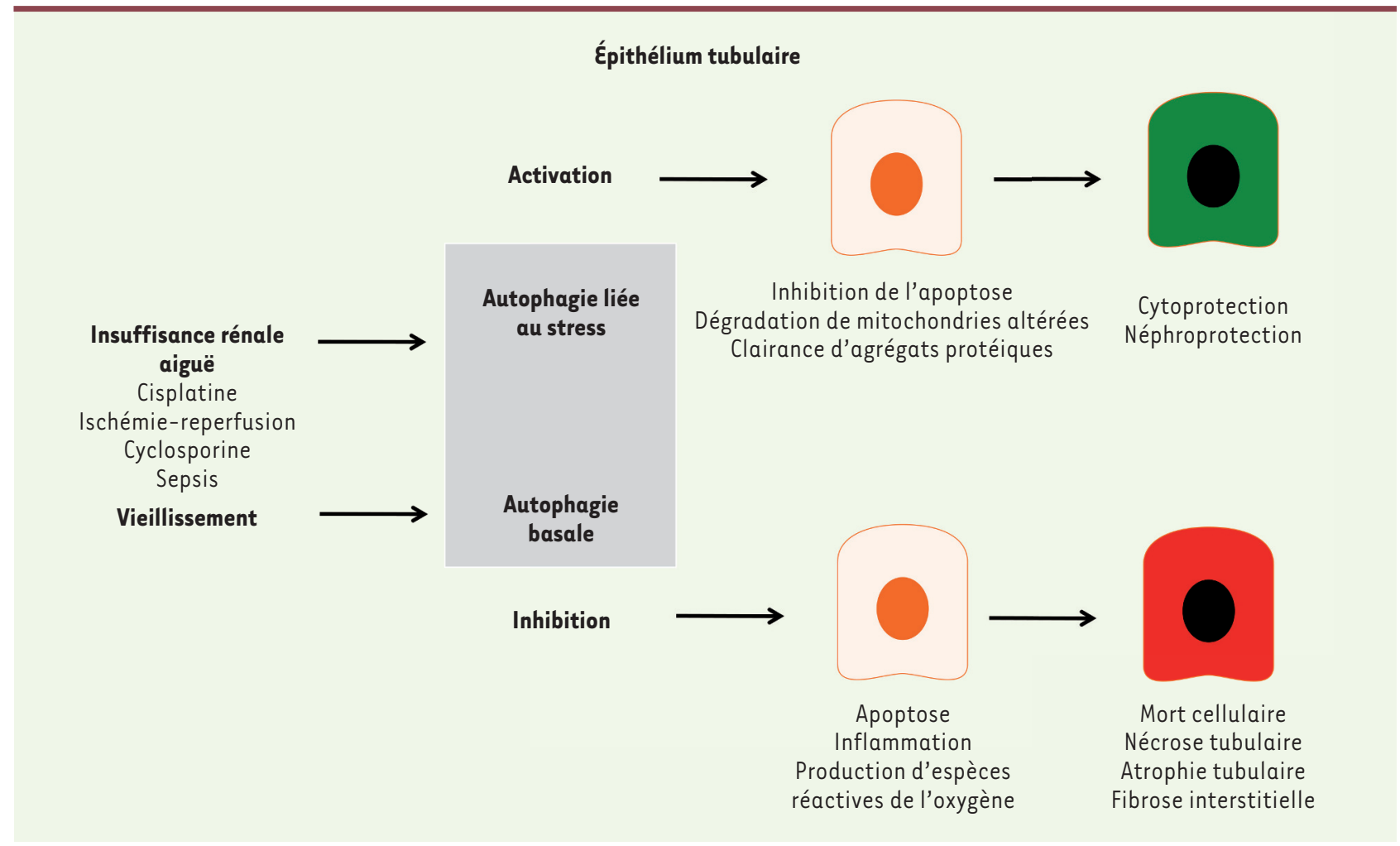

Figure 3. Fonctions de l'autophagie dans les cellules tubulaires. Les agressions rénales aiguës sont associées à une augmentation du flux autophagique, alors que l'inhibition de l'autophagie par des moyens chimiques ou génétiques aboutit à la mort cellulaire et aux altérations tissulaires. Les mécanismes possibles par lesquels l'autophagie est cytoprotectrice impliquent la dégradation de mitochondries altérées, la clairance d'agrégats protéiques, l'inhibition de l'apoptose et la réduction de la production d'espèces réactives de l'oxygène. Un déficit en autophagie augmente les lésions de nécrose tubulaire aiguë et favorise le développement de fibrose interstitielle et d'atrophie tubulaire au cours du vieillissement.

(des phagocytes non professionnels) par un mécanisme impliquant le récepteur scavenger (éboueur) KIM-1 (kidney injury molecule 1) et la molécule LC3, permettant l'élimination des débris cellulaires sans qu'il n'y ait de phénomènes inflammatoires [41]. Un déficit en KIM-l est ainsi associé à une accumulation des débris cellulaires dans la lumière tubulaire, et à une réaction inflammatoire [42]. On peut donc imaginer que l'intégrité de ce processus soit particulièrement importante pour la résolution des épisodes d'IRA, en favorisant l'élimination des débris cellulaires tubulaires tout en évitant une réaction inflammatoire.

Des liens forts apparaissent également entre homéostasie tubulaire rénale et physiologie des cils. L'impact de cette interconnexion pourrait être majeur. Les maladies rénales liées aux cils (les ciliopathies, la première d'entre elles étant la polykystose rénale) dont les mécanismes sont peu compris, ont en effet une place importante dans l'épidémiologie des maladies rénales chroniques héréditaires. Un rôle régulateur des cils sur la croissance cellulaire et la régulation de l'autophagie est maintenant démontré expérimentalement. À l'inverse, l'autophagie est connue pour réguler la ciliogenèse [43-45]. Le cil primaire est une structure sensorielle qui est exprimée à la surface des cellules. Il détecte les variations de concentrations extracellulaires en nutriments ou en facteurs de croissance, et répond aux stress mécaniques [51] $(\rightarrow)$.

La base du cil primaire est un site de recrutement des protéines ATG, notamment ATG16L1, qui facilitent le flux autophagique en réponse à des stimulus perçus par le cil comme la carence en nutriments. L'autophagie régule également la biogenèse des cils en dégradant sélectivement les protéines qui ont participé à la ciliogenèse. Ainsi, l'autophagie basale permet la dégradation de la protéine IFT20 (intraflagellar transport 20) impliquée dans la ciliogenèse, prévenant ainsi la croissance des cils. Lors d'une carence précoce en nutriments, l'autophagie va dégrader préférentiellement l'inhibiteur de la ciliogenèse, OFDl (orofacio-digital de type 1), permettant ainsi la formation des cils et, in fine, la formation d'une plateforme de recrutement de protéines ATG, avec pour conséquence une activation maximale de l'autophagie. En cas de prolongement de la carence, IFT20 sera dégradée, 
prévenant ainsi la croissance des cils et l'activation excessive de l'autophagie [43-45]. Une mutation de protéines ciliaires, les polycystines 1 et 2 , est à l'origine de la polykystose rénale qui se caractérise par le développement de kystes multiples. On sait maintenant que la croissance et la prolifération des cellules tubulaires «polykystiques », de même que le flux autophagique, sont perturbés au cours de cette pathologie [46]. Finalement, au moins théoriquement, mais aussi expérimentalement, il s'avère que les inhibiteurs de mTOR ont un intérêt dans le ralentissement de cette maladie [47]. Comme on le voit, il s'agit là de différentes pièces d'un puzzle difficile à résoudre mais il est très probable que le développement d'un modèle physiopathologique rassemblant cils, autophagie, croissance-prolifération cellulaire, et croissance des kystes pourrait être d'une grande utilité dans les années à venir.

Le compartiment tubulo-interstitiel est également la cible de lésions dues au vieillissement. Elles se caractérisent par une fibrose interstitielle et une atrophie tubulaire. Des souris dont l'épithélium tubulaire est rendu déficient en autophagie sont sujettes au développement de lésions qui sont plus sévères et plus précoces que celles présentées par des animaux sauvages [48]. Une perspective thérapeutique intéressante émerge de l'observation que le jeûne, qui active l'autophagie, protège des souris de lésions rénales liées au vieillissement, et que l'autophagie induite par la restriction calorique est néphroprotectrice [49]. Le mécanisme proposé implique l'histone désacétylase SIRTI (sirtuin 1), activée lors d'une restriction calorique (et par le resvératrol, une molécule antioxydante), qui facilite le flux autophagique $[49,50]$.

\section{Conclusions et perspectives}

En dix ans, les progrès effectués dans la compréhension des liens mécanistiques entre autophagie et physiopathologie des maladies rénales ont été majeurs. Ils ont ouvert des voies de recherche florissantes et des concepts innovants : résolution de I'IRA et de la transition vers la MRC; mécanismes des ciliopathies et croissance cellulaire ; vieillissement tissulaire. Tous ces processus impliquent l'autophagie. D'autres pistes sont encore peu explorées, notamment dans le cadre de maladies rénales inflammatoires, où l'autophagie, compte tenu de son rôle dans la régulation de l'immunité innée et adaptative, doit certainement jouer un rôle important. Sur le plan technique, il est désormais important de développer des outils et des biomarqueurs permettant de mesurer le flux autophagique chez l'homme. Ce besoin est d'autant plus nécessaire que, dans la perspective thérapeutique de cibler l'autophagie, il est indispensable que des marqueurs d'efficacité pharmacodynamique soient disponibles. Cette étape ultime, qui consistera à moduler l'autophagie chez l'homme à des fins thérapeutiques, permettra de développer des molécules ciblant spécifiquement ce processus. $\diamond$

\section{SUMMARY}

Autophagy in the kidney

Autophagy is a highly conserved, physiological, catabolic process, involving the lysosomal degradation of cytosolic components, inclu- ding macromolecules (such as proteins and lipids) and cytosolic organelles. Autophagy is believed to be essential for the maintenance of cellular homeostasis, for a number of fundamental biological activities, and an important component of the complex response of cells to multiple forms of stress. Autophagy is involved in the pathogenesis of a number of clinically important disorders but, until recently, little was known about its connection to kidney diseases. However, there is now growing evidence that autophagy is specifically linked to the pathogenesis of important renal diseases such as acute kidney injury, diabetic nephropathy and polycystic kidney disease. However, an understanding of the precise role of autophagy in the course of kidney diseases is still in its infancy. The review points out areas of particular interest for future research, and also discusses the importance of such information on whether the pharmacologic agents that modulate autophagy are potentially usable as novel forms of treatment for various kidney diseases. $\diamond$

\section{LIENS D'INTÉRÊT}

L'auteur déclare n'avoir aucun lien d'intérêt concernant les données publiées dans cet article.

\section{RÉFÉRENCES}

1. Chawla LS, Eggers PW, Star RA, Kimmel PL. Acute kidney injury and chronic kidney disease as interconnected syndromes. N Engl J Med 2014 ; 371 : 58-66.

2. Inagi $R$, Ishimoto $Y$, Nangaku M. Proteostasis in endoplasmic reticulum: new mechanisms in kidney disease. Nat Rev Nephrol 2014 ; 10 : 369-78.

3. Ericsson JL. Studies on induced cellular autophagy. II. Characterization of the membranes bordering autophagosomes in parenchymal liver cells. Exp Cell Res $1969 ; 56: 393-405$.

4. Ericsson JL. Studies on induced cellular autophagy. I. Electron microscopy of cells with in vivo labelled lysosomes. Exp Cell Res 1969 ; 55 : 95-106.

5. Pfeifer U. Morphological aspects of intracellular protein-degradation: autophagy. Acta Biol Med Ger 1981 ; 40 : 1619-24.

6. Pfeifer U, Scheller H. A morphometric study of cellular autophagy including diurnal variations in kidney tubules of normal rats. J Cell Biol 1975; 64 : 608-21.

7. Berkenstam A, Ahlberg J, Glaumann H. Isolation and characterization of autophagic vacuoles from rat kidney cortex. Virchows Arch B Cell Pathol Incl Mol Pathol $1983 ; 44: 275-86$.

8. Schiebler TH, Danner KG. The effect of sex hormones on the proximal tubules in the rat kidney. Cell Tissue Res $1978 ; 192: 527-49$.

9. Pfeifer U, Warmuth-Metz M. Inhibition by insulin of cellular autophagy in proximal tubular cells of rat kidney. Am J Physiol $1983 ; 244$ : ع109-14.

10. Bahro M, Gertig G, Pfeifer U. Short-term stimulation of cellular autophagy by furosemide in the thick ascending limb of Henle's loop in the rat kidney. Cell Tissue Res 1988 ; 253 : 625-9.

11. Hendil KB. Autophagy of metabolically inert substances injected into fibroblasts in culture. Exp Cell Res 1981 ; 135 : 157-66.

12. Shelburne JD, Arstila AU, Trump BF. Studies on cellular autophagocytosis. The relationship of autophagocytosis to protein synthesis and to energy metabolism in rat liver and flounder kidney tubules in vitro. Am J Pathol $1973 ; 73: 641-70$.

13. Bijian K, Cybulsky AV. Stress proteins in glomerular epithelial cell injury. Contrib Nephrol $2005 ; 148: 8-20$.

14. Cybulsky AV. The intersecting roles of endoplasmic reticulum stress, ubiquitin- proteasome system, and autophagy in the pathogenesis of proteinuric kidney disease. Kidney Int 2013; $84: 25-33$. 


\section{RÉFÉRENCES}

15. Imasawa T, Rossignol R. Podocyte energy metabolism and glomerular diseases. Int J Biochem Cell Biol $2013 ; 45: 2109-18$.

16. Hartleben B, Godel M, Meyer-Schwesinger C, et al. Autophagy influences glomerular disease susceptibility and maintains podocyte homeostasis in aging mice. J Clin Invest 2010 ; 120 : 1084 96.

17. Bechtel W, Helmstadter M, Balica J, et al. Vps34 deficiency reveals the importance of endocytosis for podocyte homeostasis. J Am Soc Nephrol 2013 ; 24 : 727-43.

18. Bechtel W, Helmstadter M, Balica J, et al. The class III phosphatidylinositol 3-kinase PIK3C3/VPS34 regulates endocytosis and autophagosome-autolysosome formation in podocytes. Autophagy $2013 ; 9: 1097-9$.

19. Mehta OH, Norheim G, Hoe JC, et al. Adjuvant effects elicited by novel oligosaccharide variant of detoxified meningococcal lipopolysaccharides on Neisseria meningitidis recombinant PorA protein: a comparison in mice. PLoS One 2014 ; 9 : ell15713.

20. Kawakami T, Gomez IG, Ren S, et al. Deficient autophagy results in mitochondrial dysfunction and FSGS. J Am Soc Nephrol $2015 ; 26$ : 1040-52.

21. Weindel CG, Richey LJ, Bolland S, et al. B cell autophagy mediates TLR7-dependent autoimmunity and inflammation. Autophagy $2015 ; 11: 1010-24$.

22. Inoki K, Mori H, Wang J, et al. mTORCl activation in podocytes is a critical step in the development of diabetic nephropathy in mice. J Clin Invest 2011 ; $121: 2181-96$.

23. Godel M, Hartleben B, Herbach N, et al. Role of mTOR in podocyte function and diabetic nephropathy in humans and mice. J Clin Invest 2011; $121: 2197-209$.

24. Ma T, Zhu J, Chen X, et al. High glucose induces autophagy in podocytes. Exp Cell Res 2013 ; 319 : 779-89.

25. Wu L, Feng Z, Cui S, et al. Rapamycin upregulates autophagy by inhibiting the mTOR-ULKI pathway, resulting in reduced podocyte injury. PLoS One 2013; 8 : e63799.

26. Kitada M, Takeda A, Nagai T, et al. Dietary restriction ameliorates diabetic nephropathy through anti-inflammatory effects and regulation of the autophagy via restoration of Sirtl in diabetic Wistar fatty (fa/fa) rats: a model of type 2 diabetes. Exp Diabetes Res $2011 ; 2011: 908185$

27. Palsamy P, Subramanian $S$. Resveratrol protects diabetic kidney by attenuating hyperglycemiamediated oxidative stress and renal inflammatory cytokines via Nrf2-Keapl signaling. Biochim Biophys Acta $2011 ; 1812$ : 719-31.

28. Sakaguchi M, Isono M, Isshiki K, et al. Inhibition of mTOR signaling with rapamycin attenuates renal hypertrophy in the early diabetic mice. Biochem Biophys Res Commun $2006 ; 340: 296-301$.

29. Tanaka Y, Kume S, Kitada M, et al. Autophagy as a therapeutic target in diabetic nephropathy. Exp Diabetes Res $2012 ; 2012$ : 628978.

30. Zhang MZ, Wang Y, Paueksakon P, Harris RC. Epidermal growth factor receptor inhibition slows progression of diabetic nephropathy in association with a decrease in endoplasmic reticulum stress and an increase in autophagy. Diabetes $2014 ; 63: 2063-72$.

31. Yadav A, Vallabu S, Arora S, et al. ANG II promotes autophagy in podocytes. Am J Physiol Cell Physiol 2010 ; 299 : C488-96.

32. Dong $\mathrm{C}$, Zheng $\mathrm{H}$, Huang $\mathrm{S}$, et al. Heme oxygenase- 1 enhances autophagy in podocytes as a protective mechanism against high glucose-induced apoptosis. Exp Cell Res 2015 ; 337 : 146-59.

33. Lenoir 0 , Jasiek M, Henique $C$, et al. Endothelial cell and podocyte autophagy synergistically protect from diabetes-induced glomerulosclerosis. Autophagy $2015 ; 11$ : 1130-45.

34. Ding Y, Choi ME. Regulation of autophagy by TGF-beta: emerging role in kidney fibrosis. Semin Nephrol $2014 ; 34: 62-71$
35. Chiang CK, Wang CC, Lu TF, et al. Involvement of endoplasmic reticulum stress, autophagy, and apoptosis in advanced glycation end productsinduced glomerular mesangial cell injury. Sci Rep 2016 ; 6 : 34167.

36. Kaushal GP, Shah SV. Autophagy in acute kidney injury. Kidney Int 2016; 89 : 779-91.

37. Fougeray S, Pallet N. Mechanisms and biological functions of autophagy in diseased and ageing kidneys. Nat Rev Nephrol 2015 ; 11 : 34-45.

38. Li L, Zepeda-Orozco D, Black R, Lin F. Autophagy is a component of epithelial cell fate in obstructive uropathy. Am J Pathol $2010 ; 176: 1767-78$.

39. Ding Y, Kim S, Lee SY, et al. Autophagy regulates TGF-beta expression and suppresses kidney fibrosis induced by unilateral ureteral obstruction. J Am Soc Nephrol $2014 ; 25: 2835-46$

40. Kim SI, Lee SY, Wang Z, et al. TGF-beta-activated kinase 1 is crucial in podocyte differentiation and glomerular capillary formation. J Am Soc Nephrol $2014 ; 25$ : 1966-78.

41. Brooks CR, Yeung MY, Brooks YS, et al. KIM-1-/TIM-1-mediated phagocytosis links ATG5-/ULKI-dependent clearance of apoptotic cells to antigen presentation. EMBO J $2015 ; 34: 2441-64$.

42. Yang L, Brooks CR, Xiao S, et al. KIM-1-mediated phagocytosis reduces acute injury to the kidney. J Clin Invest 2015; $125: 1620$-36.

43. Pampliega 0 , Orhon I, Patel B, et al. Functional interaction between autophagy and ciliogenesis. Nature $2013 ; 502$ : 194-200.

44. Orhon I, Dupont N, Zaidan M, et al. Primary-cilium-dependent autophagy controls epithelial cell volume in response to fluid flow. Nat Cell Biol 2016; $18: 657-67$.

45. Tang Z, Lin MG, Stowe TR, et al. Autophagy promotes primary ciliogenesis by removing OFDI from centriolar satellites. Nature 2013 ; $502: 254-7$.

46. Belibi F, Zafar I, Ravichandran K, et al. Hypoxia-inducible factor-lalpha (HIF-lalpha) and autophagy in polycystic kidney disease (PKD). Am J Physiol Renal Physiol 2011 ; 300 : F1235-43.

47. Shillingford JM, Murcia NS, Larson CH, et al. The mTOR pathway is regulated by polycystin-1, and its inhibition reverses renal cystogenesis in polycystic kidney disease. Proc Natl Acad Sci USA 2006 ; 103 : 5466-71.

48. Liu S, Hartleben B, Kretz 0 , et al. Autophagy plays a critical role in kidney tubule maintenance, aging and ischemia-reperfusion injury. Autophagy $2012 ; 8: 826-37$

49. Kume $\mathrm{S}$, Uzu T, Horiike $\mathrm{K}$, et al. Calorie restriction enhances cell adaptation to hypoxia through Sirtl-dependent mitochondrial autophagy in mouse aged kidney. J Clin Invest $2010 ; 120: 1043-55$.

50. Kitada M, Kume S, Koya D. Role of sirtuins in kidney disease. Curr Opin Nephrol Hypertens $2014 ; 23: 75-9$

51. Laclef $C$ (coordonné par). Cils primaires et ciliopathies. Med Sci (Paris) $2014 ; 30: 955-1046$

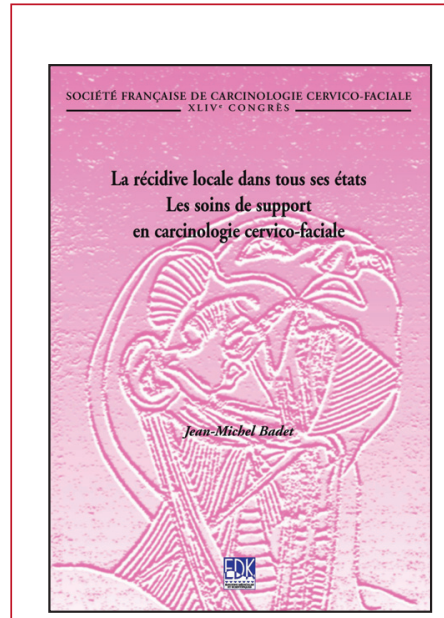

ISBN : 978-2-8425-4174-3 274 pages

\section{Bon de commande}

À retourner à EDP Sciences, 109, avenue Aristide Briand - 92541 Montrouge Cedex Tél. : 0141177405 - Fax : 0149850345 - E-mail : francois.flori@edpsciences.org

NOM : Prénom

Adresse :

Code postal :

Ville :

Pays :

Fonction :

Je souhaite recevoir l'ouvrage La récidive locale dans tous ses états - Les soins de support en carcinologie cervico-faciale : $35 €+3 €$ de port $=\mathbf{3 8} €$ TTC

en ............... exemplaire, soit un total de ..................................... €

$\square$ Par chèque, à l'ordre de EDP Sciences

$\square$ Par carte bancaire : $\square$ Visa $\square$ Eurocard/Mastercard

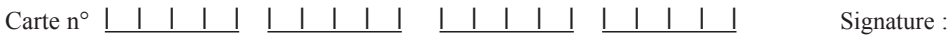

Date d'expiration: $\quad \underline{1}|\mathrm{l}| \mathrm{|} \mid$

$\mathrm{N}^{\circ}$ de contrôle au dos de la carte :

TIRÉS À PART

N. Pallet 